\title{
LES RECHARGEMENTS DE PLAGES
}

\author{
S. MANOUJIAN \\ Man Conseils - 94000 CRÉTEIL
}

\section{Summary}

Since several decades, one has been observing a graduated degradation of sandy coast-ines, such as an intensive erosion of beaches.

Growing urbanization intensified an economical aspect of this problem and led to costly, often unsightly solutions.

The "fill" of beaches is :

- to make up the unsteadiness of a coastline caused by a natural erosion or some human operations (works, sand borrowings, etc ...),

- or even to create an artificial beach.

This "fill" method applied gives the advantage of not modifying anything in the environment and avoids consequences on near installations.

Yet stabilization works can:

- either be very simple, as far as direct fill on beaches is concerned.

- or very complicated, when fill is to be connected with works such as breakwaters, jetties ...

Fill materials must answer some granulometric criteria, available on the site.

Besides earthly sediments and quarry crushed materials, sea-sands are also used, dredged from near areas then thrown up by various means.

Costs of these operations vary according to local conditions and used methods. It may go from 10 to 50 FF per cubic meter of thrown up materials. Prices of follow-up (if necessary) are the same as for usual works.

As a matter of fact, we can often settle maintenance and improvement works of access channels and port areas covered with sand at the same time as beach fills. The increase in value of dredging can be measured from throwing up installations or increasing run of dredges. Compared to our wasted coastline, even to tourist uses, this extra cost is low chiefly if one remembers that every year $800 \mathrm{~km}$ of our coasts move one meter backwards.

\section{Résumé}

Depuis plusieurs decennies, on assiste d une dégradation progressive des littoraux sableux sous forme d'une érosion intensive des plages.

Le développement de l'urbanisation a intensifié l'aspect économique de ce problème et conduit a mettre en place des solutions de defenses onereuses souvent inesthétiques.

Les rechargements de plages ont pour but de compenser un déséquilibre du littoral provoqué par une érosion naturelle ou résultant d'intervention humaines fouvrages, emprunts de sable, etc...), voire de creer une plage artificielle.
Ce procédé présente l'avantage de ne pas modifier l'environnement et d'éviter les répercussions sur les installations limitrophes.

Les travaux de stabilisation peuvent cependant aller :

- du plus simple : rechargements directs sur les plages,

- au plus complexe : rechargements associés d̀ des ouvrages (brise-lames, épis, ...).

Les materiaux de rechargement doivent répondre a certains critères granulométriques liés à leur disponibilité sur le site. Outre les sédiments terrestres, sont également utilisés les sables marins dragués puis rejetés par divers moyens et, à défaut, des matériaux de carrière concassés.

Les coûts de ces interventions varient selon les conditions locales et les méthodes utilisables. On peut estimer 10 a $50 \mathrm{~F}$ le $\mathrm{m}^{3}$ de matériaux rejetés, les prix des ouvrages d"accompagnement (s'il y a lieu) étant ceux des ouvrages classiques (en moyenne $10000 \mathrm{Frs}$ le mètre lineaire).

Dans le même ordre d'idée, les produits de dragages d'élements granuleux a l'entrée d'un port ou d'un chenal devraient être rejetés sur les plages limitrophes soit d l'estran soit par petits fonds ou stockés en vue de leur utilisation ultérieure au lieu d'être disposés au large dans des profondeurs ou ils ne peuvent plus remonter vers la côte.

\section{I - CONSIDÉRATIONS GÉNÉRALES}

\section{1 - Détermination de la granulométrie des} sables de rechargement

Pour que des matériaux déposés dans un site subsistent sous forme de plage artificielle, il faut rechercher une situation telle que les agents naturels aient moins de prise sur les apports artificiels que sur les sédiments in situ. Cette remarque confirme le fait que la plage peut atteindre une stabilité satisfaisante lorsque les matériaux d'apport sont plus gros que les matériaux en place, la pente artificielle étant alors supérieure à la pente naturelle des fonds dans l'état d'équilibre du profil. Si le matériau d'apport est trop fin, il tendra à être dispersé vers le large et la pente de la plage diminuera.

Les caractéristiques du matériau de rechargement seront définies en comparant la distribution granulométrique de ses particules à celle de la plage naturelle et l'on devra éviter que des éléments plus fins d'apport ne soient introduits. Pour préciser le pourcentage des éléments d'apport qui restera en place, Krumbein a proposé une formule basée sur la notion de distribution granulométrique des matériaux.

Elle montre que les quantités à apporter sont fonction du rapport entre les diamètres des grains constituant la plage initiale et ceux des grains constituant les apports artificiels. On peut admetre que : 
- si le matériau d'emprunt est plus gros que le matériau naturel, le volume nécessaire sera de l'ordre de 1,5 fois le volume estimé.

- si le matériau d'emprúnt est plus fin que le matériau naturel, le volume à apporter pourra être 5 à 10 fois le volume théorique sans que l'on soit certain d'atteindre un état stable de la plage par suite d'un départ des sédiments vers le large.

A noter que pour pallier le manque de matériau grossier permettant d'obtenir de bons résultats, il est possible d'utiliser du concassé de carrière en remplacement du sable naturel (exemple : plage du Larvotto à Monaco (plage du Prado à Marseille).

1.2 - Techniques d'apports des matériaux de rechargement

\subsection{1 - Reconstitution d'une plage}

Sources d'apports

Lorsqu'il s'agit de reconstituer une plage ayant subi une grande déperdition de sable, il est possible d'avoir recours à des rechargements en sédiments :

- dragués en mer à des profondeurs suffisantes.

La profondeur acceptable de prélèvement en mer peut être estimée en fonction des études réalisées sur les stabilités des souilles d'extraction et l'on peut fixer les fonds de $-20 \mathrm{~m}$ sous le zéro des basses mers, dans les zones les plus exposées du littoral français, comme limite à partir de laquelle les extractions n'auront plus d'effet sur l'équilibre sédimentaire. [1]
- prélevés dans des zones diaecumulation susceptibles d'être néfastes pour la navigătion (exemple : chenal d'accès de l'Adour),

- extraits de flèches sableuses ou des plages excessivement engraissées,

- dragués en rivière,

- extraits de carrières (graviers).

\section{Techniques d'apports}

Plusieurs techniques peuvent être envisagées :

- lutilisation de conduites de refoulement direct entre le lieu de prélèvement et celui du rejet, dont la distance ne peut pas dépasser 2 à $3 \mathrm{~km}$ sans installation intermédiaire (fig. 1a),

- le transbordement par la drague :

* soit jusqu'au site à réalimenter par clapage aussi près que possible de l'estran

* soit, jusqu'à une souille de mise en dépôt intermédiaire réalisée à l'intérieur du port (par exemple), les matériaux seront ultérieurement repris par une conduite de refoulement direct sur la côte (fig. $1 \mathrm{~b}$ et $1 \mathrm{c}$ ),

- le transport terrestre par camions après reprise par extracteurs, dragline, etc ... sur les plages ou dans les zones sédimentées accessibles (tombolos) (fig. 1d).

A noter que lorsqu'il s'agit d'apports prélevés au large, l'efficacité est moindre ou du moins temporaire, vu la trop grande finesse caractérisant fréquemment les sédiments du large. a - REFOULEMENT DIRECT A L'ESTRAN

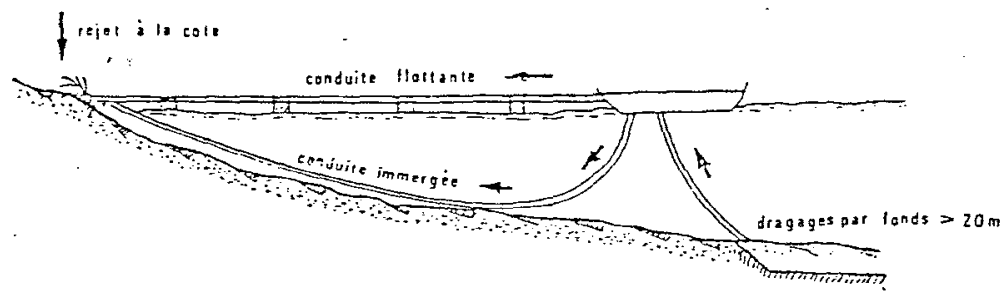

c - DEVERSEMENT A QUAI ET REPRISE PAR POMPAGE

\section{b - CLAPAGE PAR PETTTS FONDS}

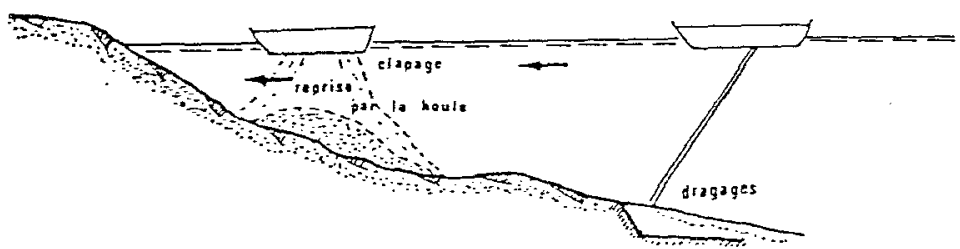

c - BY PASS AVEC OU SANS CREATION DE TOMBOLO

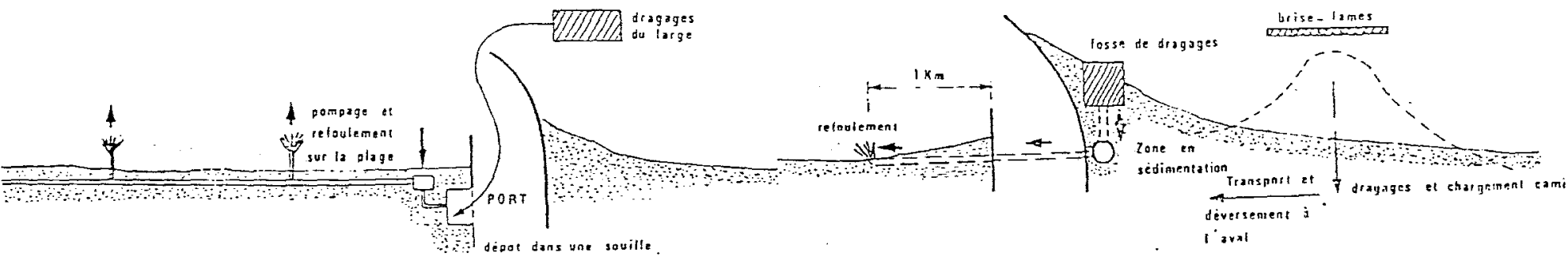

Fig. 1 : Techniques d'apports des matériaux de rechargement de plage 


\subsection{2 - Rétablissemențd'un transit littoral}

Lorsqu'il s'agit de rétablir un transit littoral et de maintenir ainsi l'équilibre de plages menacées par l'arrêt des sédiments le long d'une jetée, d'un port ou d'un chenal d'accès, on peut utiliser un dispositif de "by-pass". Le matériau déposé le long de l'ouvrage portuaire est repris par différents procédés et redéposé à laval sur l'estran pour rétablir le transit. La reprise des sédiments pourra se faire par une drague en mer avec refoulement à terre à l'aide d'une conduite et de stations de pompages intermédiaires mais un tel dispositif ne peut fonctionner en période de forte houle; c'est cependant à ce moment que le transit littoral est le plus important. On peut améliorer le dispositif en piégeant le sable, avant son accumulation le long de l'ouvrage portuaire, à l'abri d'un brise-lames ; le dépôt sera alors pompé à poste fixe et refoulé par conduite ou extrait par voie terrestre (extracteur, drag-line, etc ...).

\subsection{3 - Entretien des plages rechargées}

Dans de nombreux cas, l'effet spectaculaire obtenu immédiatement après rechargement s'amenuise au cours du temps si des apports périodiques ne sont pas effectués et ceci d'autant plus fréquemment que les sédiments sont fins, la perte vers le large étant liée au degré de finesse.

\section{2 - DÉVERSEMENT DES MATÉRIAUX D'APPORT SUR LES PLAGES}

\section{1 - Mode de déversement}

Pour réalimenter une plage en érosion, il est souhaitable de déverser les matériaux directement sur le haut-estran en laissant à la houle le soin de répartir les matériaux et de modeler le profil.

Ces apports peuvent être réalisés soit par la méthode classique de transport intermittent à l'aide de camions, soit par la mêthode plus onéreuse mais plus efficace décrite précédemment, utilisant les apports amenés par conduites disposées le long du littoral avec des stations de relais si nécessaire (fig. $1 c$ et $1 \mathrm{~d}$ ).

Bien que l'inconvénient de ce dernier procédé de rechargement soit son prix excessif, le $\mathrm{m}^{3}$ de sable refoulé étant de l'ordre de $50 \mathrm{~F} / \mathrm{m}^{3}$ suivant l'importance et les difficultés de chantiers, de nombreux pays n'hésitent pas à engager de telles dépenses pour sauver leur littoral.

\section{2 - Exemples de rechargement de plages à} l'estran

2.2.1 - Dans les exemples généraux de rechargement de plages en érosion ou création de nouvelles plages, nous pourrons citer :

* Simples apports et déversements par voie terrestre pour lutter contre l'érosion

- sur la plage de Carnon, un simple rechargement de $19000 \mathrm{~m}^{3}$ de sable, extrait du port, associé à deux petits brise-lames disposés par fond de $-1 \mathrm{~m}$ au droit de deux casiers constitués par des épis a permis de favoriser la formation de tombolos,

- à Criel, des essais de réengraissement à l'aide de galets prélevés au Tréport ont donné des résultats satisfaisants,

- à Anglet, [4 et 5] devant l'ampleur des phénomènes d'érosion qui menaçaient le littoral, des recherches de protection ont été effectuées, dont un premier type d'intervention qui portait sur le secteur sud de la plage d'Anglet (vue générale sur fig 2) et consistait à déverser dans les alvéoles sédimentaires créées entre des épis de 100 à $135 \mathrm{~m}$ de long de grandes quantités de sable grossier mélangé à de la madrague (30\% de gravier) prélevé à la côte au voisinage de l'embouchure de 1:Adow.

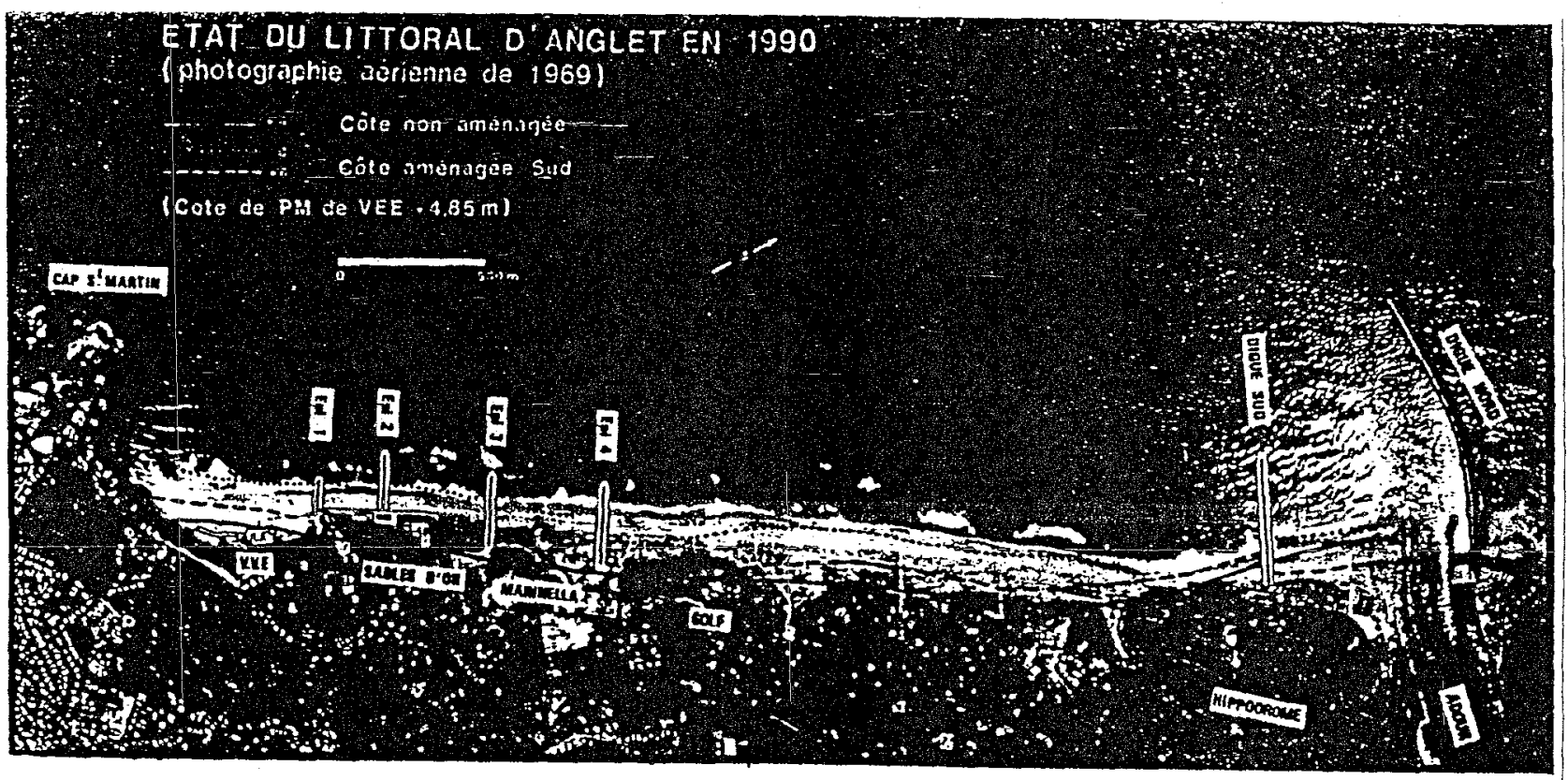

Fig. 2 : Etat du littoral d'Anglet en 1990 
Hélas le volume global déversé de 1975 à 1977 n'a pas excédé $120000 \mathrm{~m} 3$ '(très inférieur à celui préconisé). En outre, la granulométrie était trop faible et les sédiments fins ont été dispersés vers le large. L'engraissement de la plage n'a donc été que temporaire. Des rechargements périodiques de ce type auraient été nécessaires pour éviter le recul inexorable de la microfalaise et l'abaissement du niveau de plage,

- dans la baie de Cavalaire où l'on peut comparer, d'une part le comportement de l'alvéole ouest dans laquelle les apports sont bloqués entre le port et un épi avec constitution d'une plage attrayante, d'autre part l'érosion de l'alvéole est non rechargée,

- à la Rague une comparaison identique entre deux alvéoles protégées par des épis en $\mathrm{T}$,

- au Moulin Blanc un apport de sable grossier bloqué par un épi,

- au Prado un apport massif des graviers dans un gigantesque complexe touristique.

* Pour créer des plages artificielles

- au Mourillon le déversement d'une grande quantité de sable moyen $(200 \mu \mathrm{m})$ à l'abri d'un brise-lames.

- à Bonnegrace le même procédéentre une digue et un épi.

* Des alimentations par conduites

- sur la plage de Seagirt (New Jersey) des produits dragués au large ont été refoulés en haut de plage à raison de $250000 \mathrm{~m}^{3}$ par kilomètre de côte par une conduite posée sur le fond. Pour faciliter le travail, une barge flottante amarrée au large servait d'accostage aux dragues porteuses refoulantes,

- à Redondo Beach (Californie), le même procédé a été utilisé avec succès avec un apport de $250000 \mathrm{~m}^{3}$ prélevés par fonds de - $17 \mathrm{~m}$ sous le niveau des basses mers,

- à la Noveillard, à louest de Pornic, à l'occasion de l'édification du port de plaisance, l'extension de la plage située au nord du port a été réalisée en 1972. Cette plage de $200 \mathrm{~m}$ de long délimitée par deux épis de 70 à $100 \mathrm{~m}$ de longueur n'était pas alimentée dans les conditions océanographiques auxquelles elle était soumise. Environ $40000 \mathrm{~m} 3$ de sable provenant du dragage du port ont été déversés par rejets hydrauliques entre $+6,50$ et $+0,50 \mathrm{~m}$, les sédiments déversés ayant une granulométrie supérieure à celle du matériau en place. Les résultats obtenus pour un coût de $5 \mathrm{~F}$ le $\mathrm{m}^{3}$ environ en 1972 ont été satisfaisants.

- à Knokke en Belgique [5], au nord de Zeebrugge, la plage qui avait subi une érosion importante depuis la construction des ouyrages portuaires a été réengraissée sur $8 \mathrm{~km}$ de longueur, en déversant sur la côte plusieurs millions de $\mathrm{m}^{3}$ de sable dragué dans le port à l'aide d'une drague aspiratrice-refoulante. Les canalisations disposées depuis le poste de prélèvement jusqu'aux divers points de rejet avaient environ 28 pouces et permettaient d'evacuer directement le sable en haut de plage à $3 \mathrm{~km}$ maximum du point d'aspiration sans relais intermédiaire.

Le second type d'intervention sera un déversement par petits fonds (cf 3.2).
L'incenvénient de ce procédé est son prix prohibitif, le $\mathrm{m}^{3}$ de sáble refoulé pouvant atteindre $40 \mathrm{~F}$ le $\mathrm{m}^{3}$ en 1982 suivant l'importance et les difficultés du chantier.

2.2.2 - Des exemples plus spécifiques de rechargement de plage s'appliquent au rétablissement d'un transit littoral interrompu partiellement ou totalement par la construction d'ouvrages portuaires et provoquant nécessairement de notables érosions à l'aval des ouvrages.

- à Santa Barbara (Californie), (fig 3) la quasi totalité du transit sédimentaire s'est accumulée d'une part à l'enracinement ouest du brise-lames protégeant le port, d'autre part sous forme d'une importante flèche sableuse en extrémité d'ouvrage. Un système de by-pass, par pipeline reliant la drague qui prélevait sur ce dépôt jusqu'au point de rejet à permis de réalimenter la plage est en érosion, 4,5 millions de $\mathrm{m}^{3}$ ayant été déposés entre 1940 et 1952 . Ultérieurement, il fut décidé de prélever essentiellement les atterrissements dans le chenal d'accès par l'intermédiaire d'une drague flottante dans le double but de réalimenter la plage et de maintenir des profondeurs dans le chenal.

- à South-Lake Worth (Floride), les deux jetées construites afin de draguer un chenal d'accès au "Lake Worth" ont provoqué une accumulation importante des sédiments sur la face nord résultant d'un transit nord-sud d'environ $200000 \mathrm{~m}^{3} /$ an et par voie de conséquence un recul de la côte sud.

Un système de pompage (capacité voisine de $50 \mathrm{~m}^{3}$ de sable par heure) aspire le sable sédimenté contre la jetée nord et le refoule par un système de by-pass sur la plage nord érodée qui se reforme progressivement. Ce mode de réalimentation permet de rétablir partiellement le transit (fig. 4).

Une autre réalisation de ce type a été utilisée à Palm Beach en Floride (fig. 4).

Enfin, en France sur la côte Atlantique, un projet de ce type a été envisagé à Cap Breton, par un système d'hydro-éjecteur (fig. 5) [8].

Un tel procédé avait été envisagé sur le littoral Adour Anglet dans le double but de réduire les apports dans le chenal d'accès au port de Bayonne et de réalimenter les plages d'Anglet en érosion permanente (voir schéma d'installation projetée sur fig.7).

Son coût prohibitif n'a pas permis sa réalisation.

\section{3 - DÉVERSEMENT DES MATÉRIAUX PAR PETITS FONDS}

\section{1 - Mode de déversement}

Les produits de dragages provenant d'une zone d'accès à un port ou de l'embouchure d'une rivière peuvent être réutilisés pour alimenter une plage en déversant les produits dans la zone des petits fonds. Suivant la profondeur à laquelle les dépôts seront effectués et la nature des sédiments, les matériaux pourront remonter vers la plage ou, au contraire, être dispersés vers le large.

Ce mode d'apport qui permet de réduire les frais de mise en place des sédiments de réalimentation devra donc être très soigneusement étudié avant d'être appliqué à une grande échelle. 
Fig 4

a - STATION DE REPRISE FIXE

Lake Worth Inlet, Floride (d'après CERC)

Fig 3 - BY PASS DU TRANSIT SABLEUX Santa Barbara, Californie
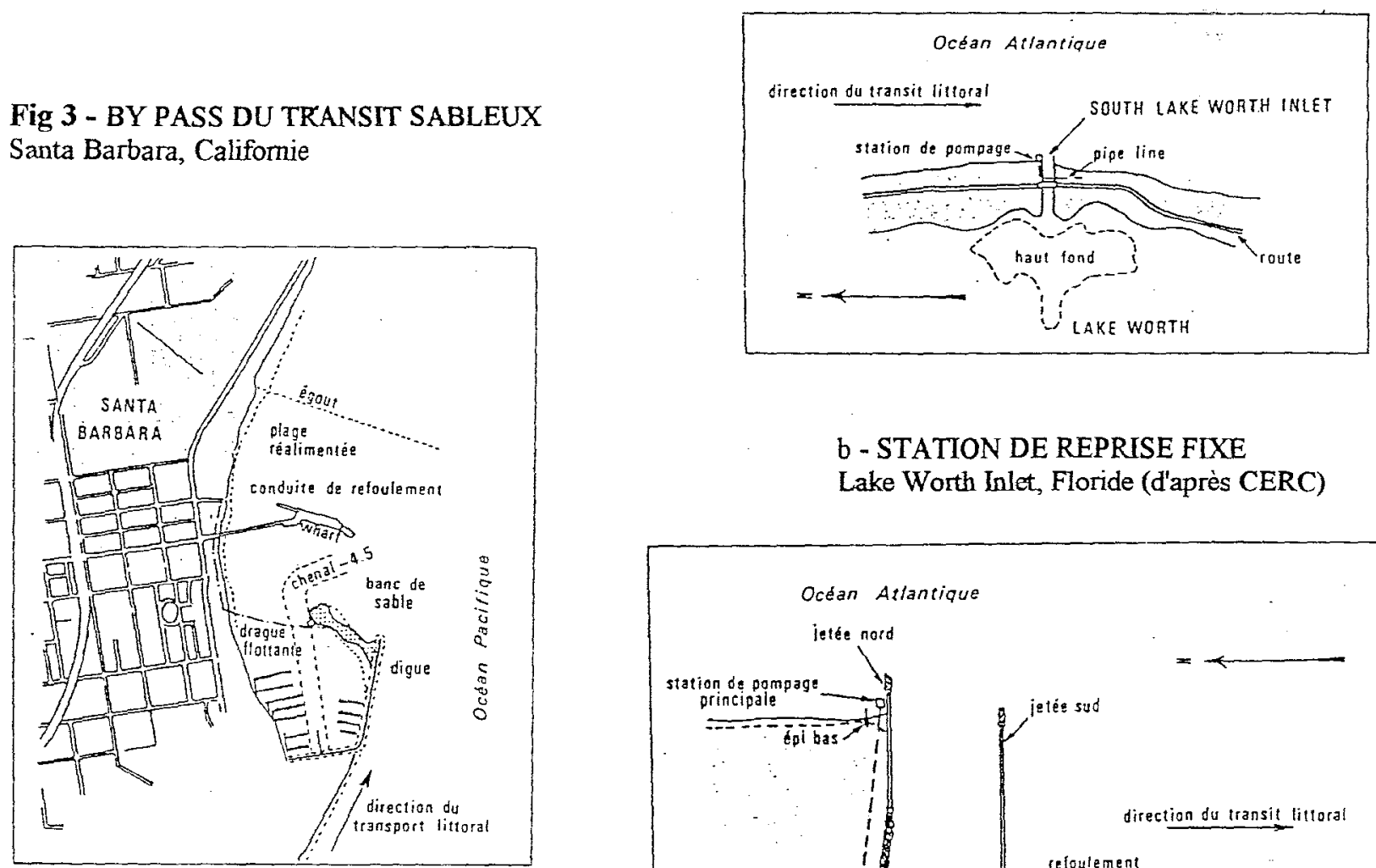

b - STATION DE REPRISE FIXE

Lake Worth Inlet, Floride (d'après CERC)
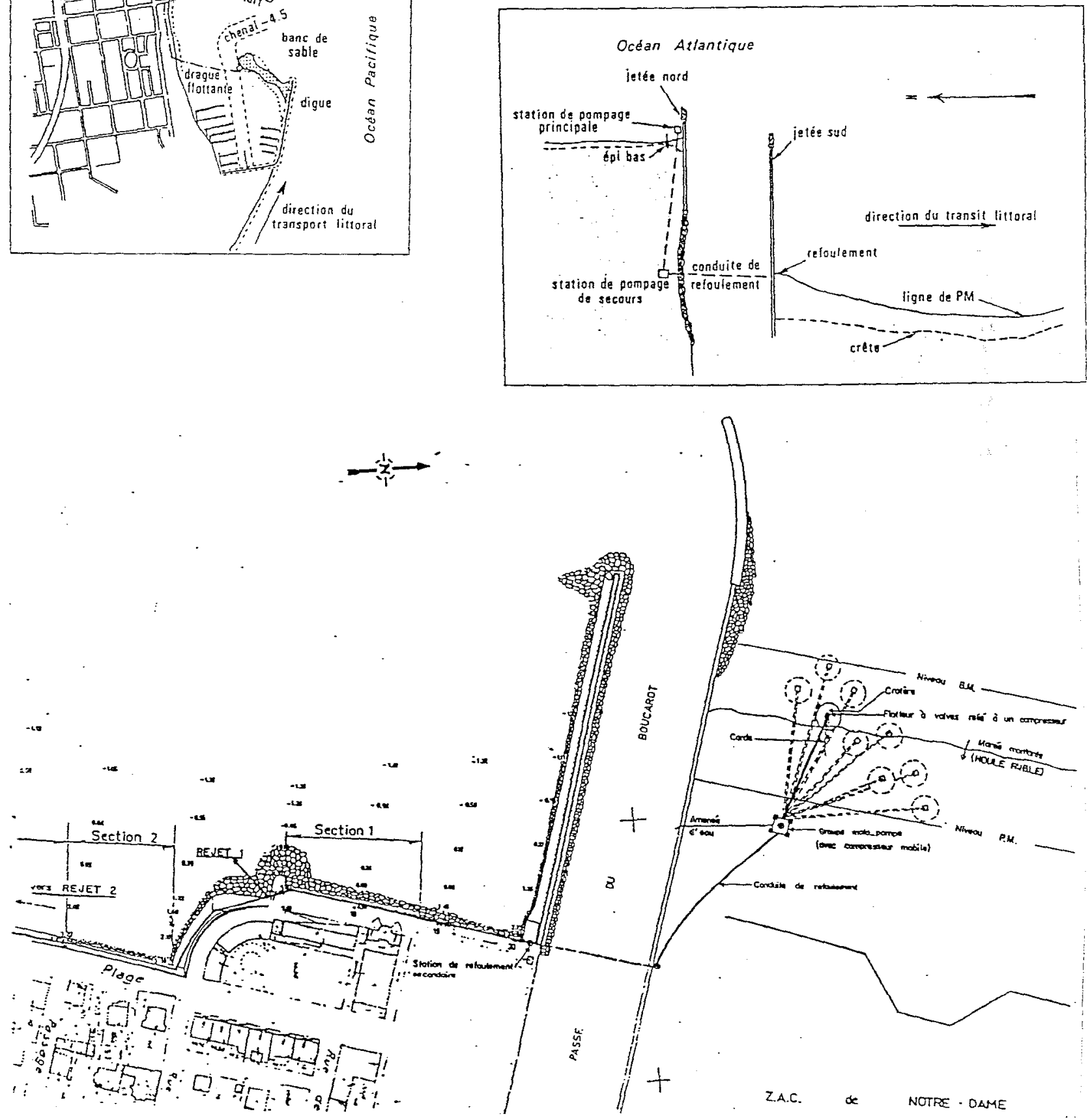

Fig 5 - TRANSFERT DE SABLE A CAPBRETON EN UTILISANT UN HYDROE.JECTEUR A BALLAST 
Nous verrons dans les exemples cités ci-après que l'efficacité du procédé dépend essentiellement des possibilités de déversements qui devront être faits aussi près que possible de l'estran, donc des moyens mis en oeuvre.

\section{2 - Exemples de déversement par petits fonds}

Diverses tentatives ont été faites aux Etats-Unis : à Long Branch (New Jersey), à Santa Barbara, à Atlantic City (New Jersey).

Les essais faits à Long Branch son t particulièrement intéressants car ils ont été suivis très attentivement. Un dépôt de sables $(D=0,3 \mathrm{~mm})$ de $2 \mathrm{~m}$ de hauteur a été fait dans des fonds de 10 à $12 \mathrm{~m}$. Pendant la période des mesures, la houle moyenne était de l'ordre de $0,5 \mathrm{~m}$ et la plus forte de $3 \mathrm{~m}$. Les périodes étaient en moyenne de 7 à $8 \mathrm{~s}$. Les divers levés bathymétriques réalisés ont montré qu'il y avait eu mouvement des matériaux (latéralement et transversalement) dans toute la zone du dépôt mais que, toutefois, il n'y avait pas eu de remontée à la côte.

Ces résultats sont analogues à ceux obtenus à Santa Barbara (Dépôts par $-6 \mathrm{~m}$ de profondeur) et à Atlantic City (dépôts entre $-4,5$ et $-7,5 \mathrm{~m}$ ).

- Le L.C.H.F. a étudié en modèle réduit et en nature les conditions de réalimentation de la plage d'Agadir (Maroc) [5] en association avec un brise-lames, par les produits de dragages provenant de la passe d'entrée au port (fig. 6).

Dans cette optique, il s'agissait de rechercher les profondeurs limites où les sédiments pouvaient être déversés avec Ie maximum d'efficacité de réengraissement de la plage sud. Celle-ci était très sous-alimentée et érodée depuis la construction du port d'Agadir qui avait entraîné un arrêt du transit littoral, se traduisant par la formation d'une flèche sédimentaire en extrémité du brise-lames principal ( $\left.400000 \mathrm{~m}^{3} / \mathrm{an}\right)$.

Dans cette zone, $75 \%$ des houles sont comprises entre 0,5 et $1,5 \mathrm{~m}$ et $3 \%$ des houles sont supérieures à $5 \mathrm{~m}$. L'influence de la profondeur de déversement des sédiments sur le maintien de la plage a été examinée en effectuant des dépôts à $-6 \mathrm{~m}$ (dépôts arasés à $-2 \mathrm{~m}$ ), à $-4 \mathrm{~m}$ (dépôts arasés à $-2,5 \mathrm{~m}$ ), entre -3 et $-1 \mathrm{~m}$, et à la cote 0 . Il a été montré que, pour que la plage puisse être réalimentée, les dépôts devaient être faits à une profondeur inférieure à $-3 \mathrm{~m}$ sous le niveau des basses mers (marnage de $4 \mathrm{~m}$ ).

Ces résultats ont été confirmés en nature comme le montre la figure 6 donnant la comparaison des fonds entre 1962 et 1972 . Les dépôts ont été faits par - $3 \mathrm{~m}$ et on peut remarquer que l'estran devant les zones de rejet s'est engraissé.

- La côte d'Anglet : Jusqu'en 1972, la totalité des sables dragués dans le chenal d'accès à l'embouchure de l'Adour étaient rejetés par profondeur de $-20 \mathrm{~m}$ sous forme d'un énorme dépôt très faiblement repris par la houle et remonté vers la côte.

Le volume extrait, très important $\left(20\right.$ millions de $\mathrm{m}^{3}$ entre 1945 et 1986) provenait en grande partie du transit (sud-nord et représentait un déficit sédimentaire certain pour tout le littoral situé entre le Cap Saint-Martin et l'embouchure, caractérisé par un recul spectaculaire de la côte d'Anglet menaçant les immeubles riverains. La nécessité de lutter contre cette érosion a conduit à préconiser une réalimentation des petits fonds afin de suppléer à l'insuffisance du rechargement d'estran.

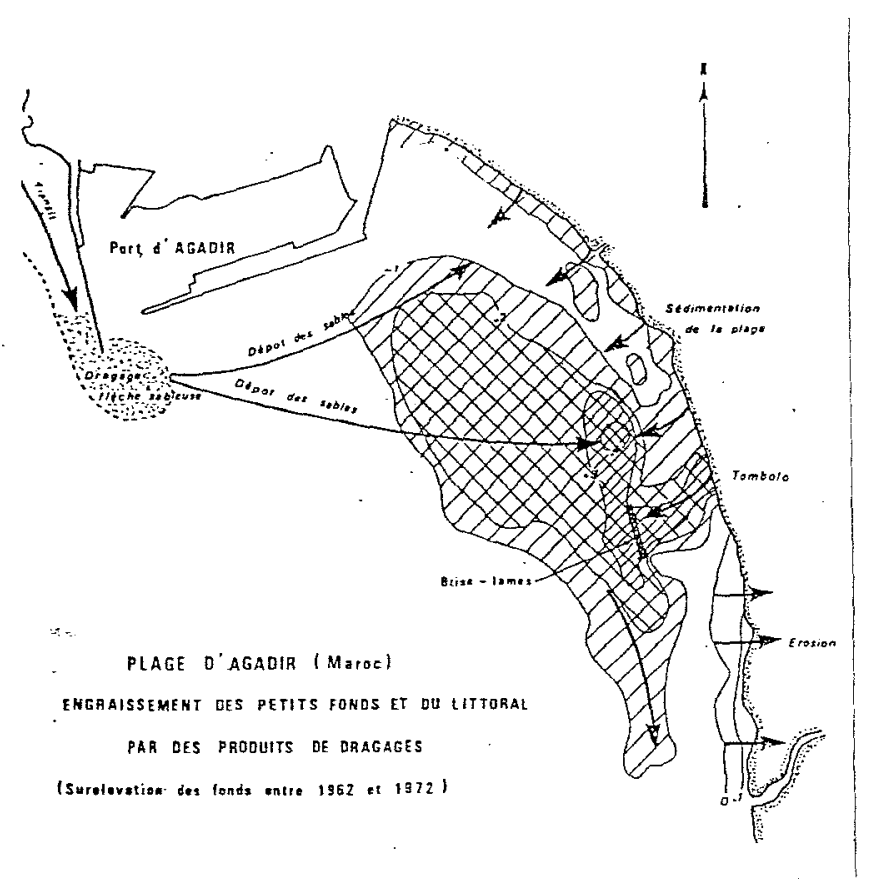

Fig. 6 : Réalimentation de la plage d'Agadir

De même que pour Agadir, le L.C.H.F. a effectué des essais en modèle réduit qui ont également montré la nécessité d'alimenter dans une zone aussi proche que possible de la côte.

Compte tenu des moyens mis en oeuvre, les produits de dragages ont été effectués soit par profondeurs voisines de -7 à $-10 \mathrm{~m}$, soit à l'aide d'une drague spéciale permettant de déverser très près du rivage jusqu'à -2 à $-3 \mathrm{~m}$ (fig. 7).

Ainsi sur 13 millions de $\mathrm{m}^{3}$ de sables déposés entre 1973 et 1992 sur le littoral d'Anglet (20 ans), seule $560000 \mathrm{~m}^{3}$ ont été alimentés en 1980 au voisinage de la côte selon les profondeurs préconisées par l'expérience.

L'observation des évolutions de fonds au cours du temps a montré que lorsque les déversements sont trop éloignés, il y a étalement et dispersion des sédiments, sans remontée appréciable à la côte (fig. 8 et 9 ).

Lorsque la drague atteint des profondeurs beaucoup plus faibles, on obtient une réalimentation du bas estran relativement efficace sans constater toutefois un réengraissement appréciable du haut estran.

Par contre, le profil des fonds qui était tronqué à la cote - 4 à - $5 \mathrm{~m}$ jusqu'au bas des plages tend progressivement à reprendre un profil parabolique plus proche des profils de stabilité des plages.

\section{4 - ORDRE DE GRANDEUR DES COÛTS}

Le coût des travaux à entregrendre tant en ce qui concerne les rechargements de plage proprement dits que la mise en oeuvre des ouvrages d'accompagnement, si 
Fig 7 - DEPOT DES MATERIAUX DRAGUES DANS L'ADOUR AU LARGE DE LA COTE D'ANGLET

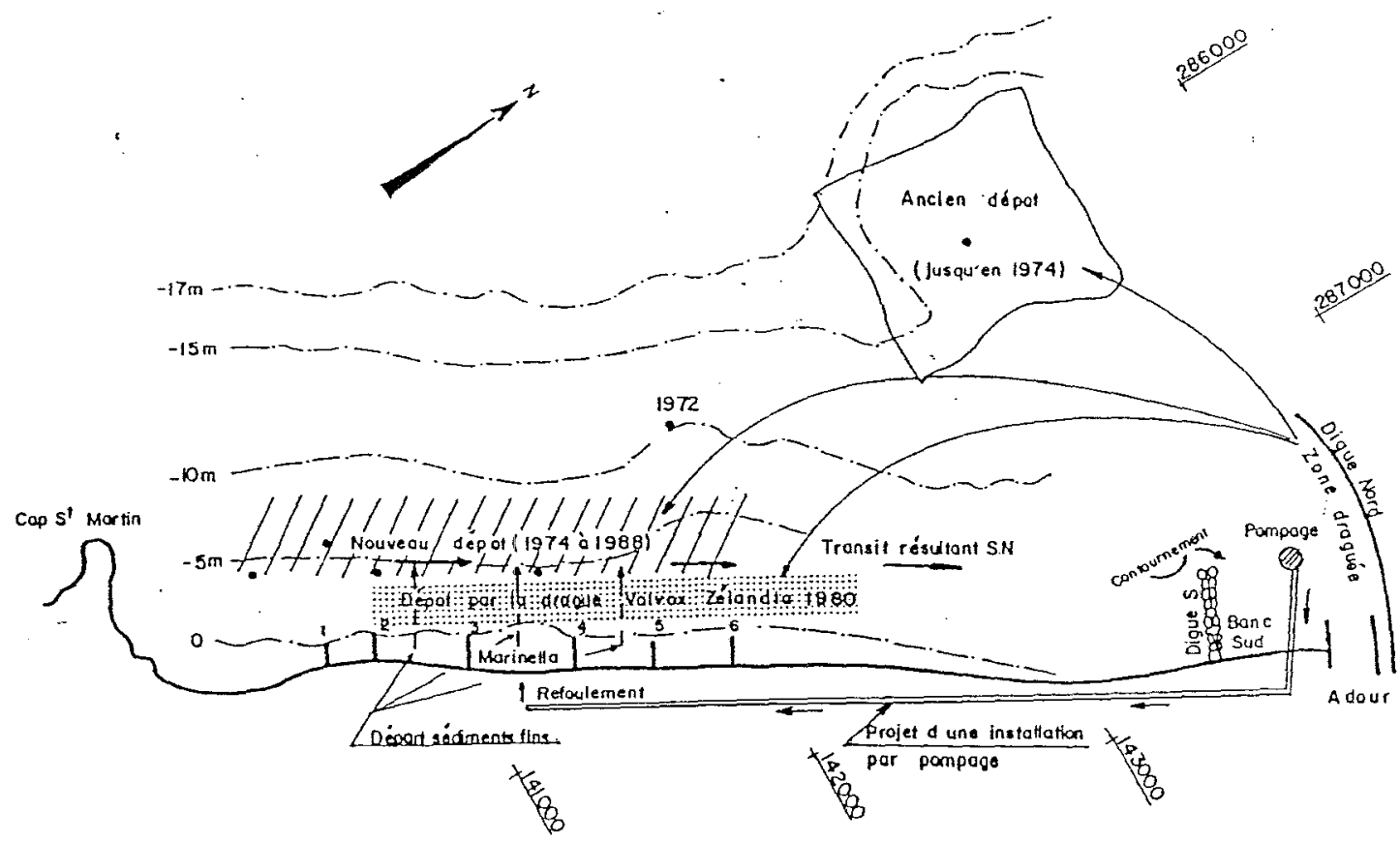

Fig 8 - SCHEMA DE LA PROGRESSION DES DEPOTS DE DRAGAGES VERS LA COTE

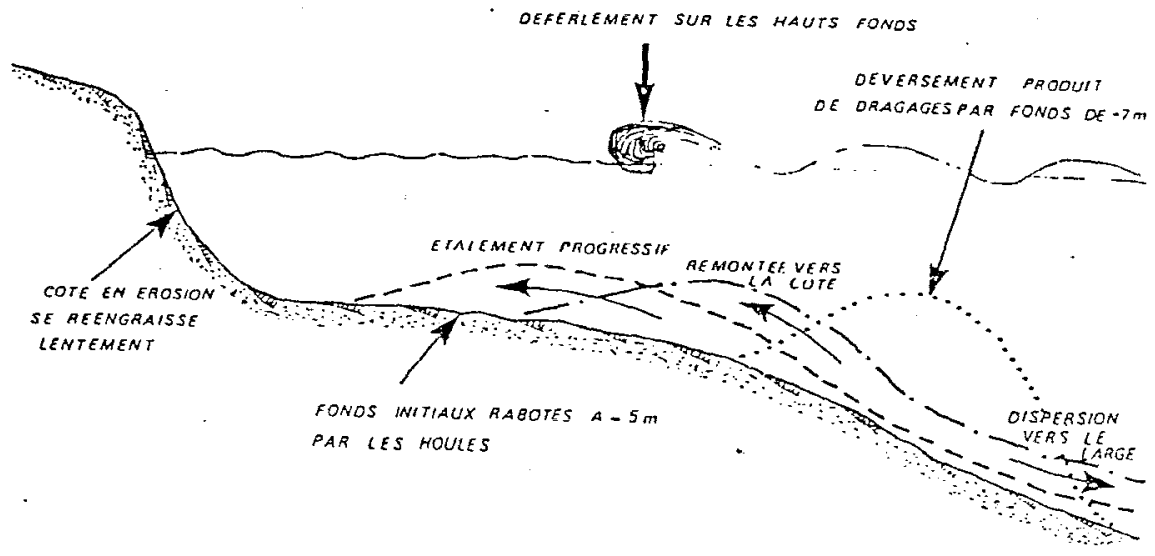

Fig 9 - REPARTITION DES ZONES D'ENGRAISSEMENT ET D'EROSION LE LONG DE LA COTE D'ANGLET AU COURS DES DERNIERES ANNEES

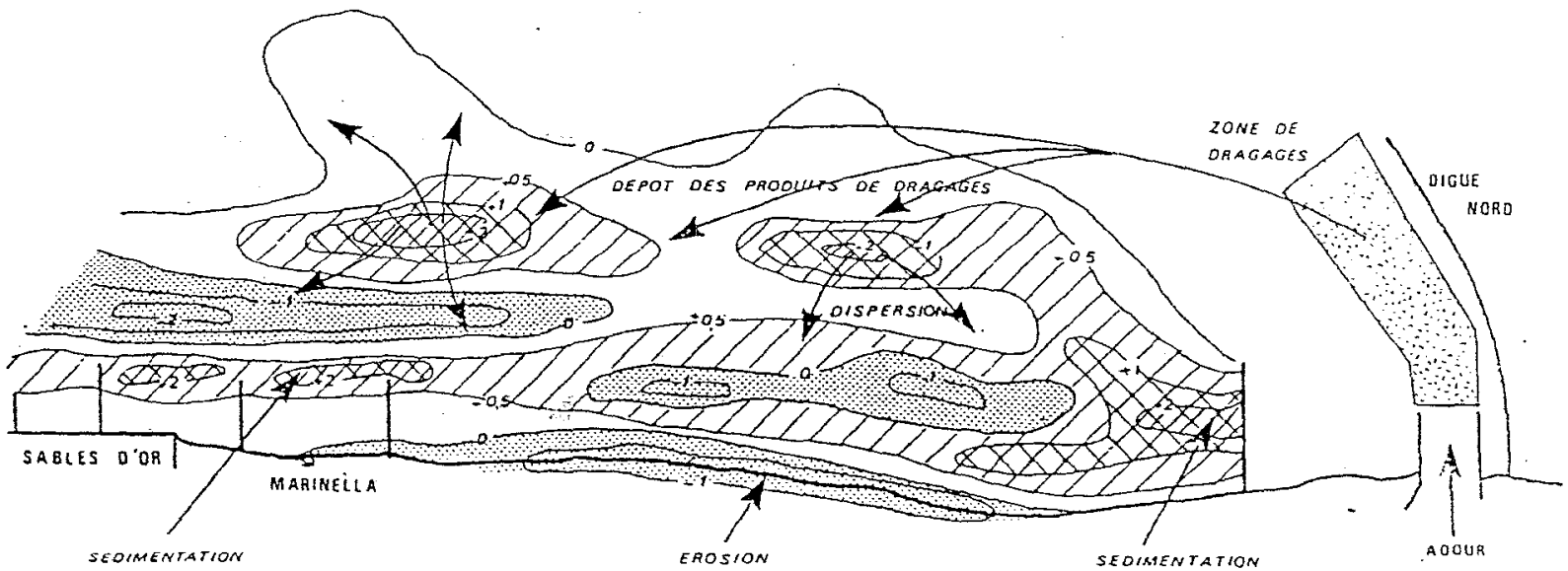


nécessaire, est un élłment non négligeable qui guidera le maître d'ouvrage darss le choix du système de défense ou d'aménagement et dans la décision qu'il prendra de faire exécuter ou non les travaux.

Par ailleurs, la connaissance de ce coût permettra la comparaison de l'investissement à effectuer et de l'enjeu à préserver bien que, dans beaucoup de cas, celui-ci soit difficilement chiffrable du fait de sa nature touristique voire écologique.

Enfin, il ne faut pas négliger les frais dentretiens périodiques qui nécessitent la mise en place d'un budget permanent.

Les coûts que nous donnons ci-après sont des valeurs moyennes (coûts unitaires en F TTC ; ces prix établis en 1989 sont à actualiser).

Ils portent sur la fourniture, le transport et la mise en oeuvre des rechargements. Pour la construction d'ouvrages d'accompagnement (perré, épis, brise-lames, etc ...), on se reportera à l'étude référencée en annexe [7].

\section{1 - Par voie terrestre}

Les prix moyens varient suivant la distance à parcourir par les camions ainsi que la nature des sédiments à mettre en place:
- pour les sables : 85 à $170 \mathrm{~F}$ le $\mathrm{m}^{3}$

- pour le concassé de carrière : 100 à $150 \mathrm{~F}$ le $\mathrm{m}^{3}$

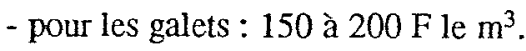

\section{2 - Par chalands (sédiments prélevés en mer)}

Le transport par chaland jusqu'à la côte est de 40 à $50 \mathrm{~F}$ le $\mathrm{m}^{3}$. A ce prix il faut ajouter le transport par camion très variable suivant la distance qui peut varier de 0 à quelques kilomètres.

\section{3 - Par voie maritime et conduite de refoulement}

Si l'on exclut l'investissement du matériel et l'entretien d'un tel système, le prix de revient est de l'ordre de 40 à $50 \mathrm{~F}$ le $\mathrm{m}^{3}$. Ceci n'est valable que pour des volumes supérieurs à $100000 \mathrm{~m}^{3}$.

4.4 - Par dragages et conduites de refoulement directement sur la plage

- En Belgique, le prix de revient du mètre cube de sable refoulé sur la plage de Knokke à l'aide de conduites
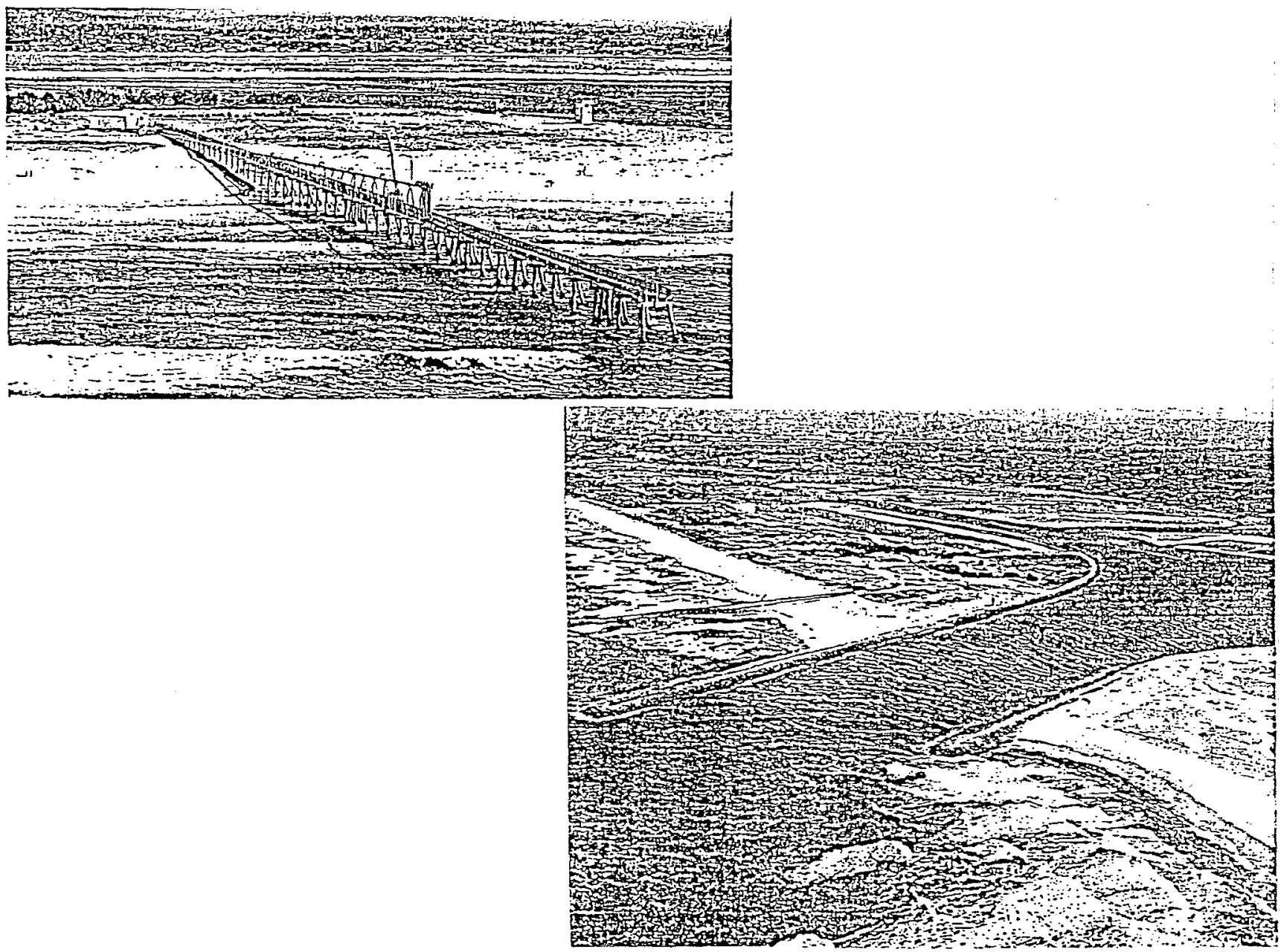

Fig. 10: Station de pompage by-pass en Australie 
permettant l'évacuation de sable depuis le point de prélèvement jusqu'au rejet ( $3 \mathrm{~km}$ environ) est voisin de $40000 \mathrm{FF}$ par mètre linéaire de plage et de $45 \mathrm{FF}$ par $\mathrm{m}^{3}$ de sable transporté.

L'entretien ultérieur des conduites est de $1000 \mathrm{FF} / \mathrm{ml}$ de plage et le prix de revient par touriste et par jour évalué à 1,5 FF.

- En Hollande, le coût de refoulement direct par conduite sur $500 \mathrm{~m}$ de distance est de $15 \mathrm{~F} \mathrm{le}^{3}$.

- A Bordeaux, ce coût atteint 20 à $30 \mathrm{~F}$ le $\mathrm{m}^{3}$.

- A Cap Breton, le projet d'installation a été chiffré à 2 millions de FF (comprenant pompes, hydroéjecteurs, conduites, etc ...) et le coût du sable transporté à $10 \mathrm{~F}$ le $\mathrm{m}^{3}$.

- En Australie, une station de grande envergure (fig 10) a été réalisée pour l'équivalent de 35 millions de FF.

\section{5 - CONCLUSIONS}

On ne peut qu'attirer l'attention sur les possibilités offertes par l'utilisation de matériaux marins, estuariens, fluviaux, ou extraits de carrière pour réalimenter des plages en voies d'érosion, reconstituer celles qui auraient disparu ou en créer de nouvelles (plages artificielles).

Cette technique de lutte contre l'érosion d'un littoral par des produits prélevés dans des secteurs situés en dehors des zones d'influence sur le littoral et déposés sur l'estran avec une granulométrie appropriée a déjà été utilisée et semble de plus en plus conseillée.

Une telle solution que nous pourrons intituler "médecine douce" n'apporte pas de préjudices à l'environnement et rétablit d'une façon naturelle un site dégradé. Elle présente des avantages certains, même si une première estimation montre qu'elle est onéreuse.

Cependant les impératifs techniques et financiers ne permettent pas toujours l'emploi d'une telle méthode.

Techniquement il faut que, selon le mode de rechargement envisagé :

- la mise en place de conduites puis leur entretien ultérieur soit possible,

- Ies dragues appropriées au déversement par petits fonds soient disponibles,

- le cheminement des camions par voie terrestre n'entrave pas la circulation citadine, etc ...

En fait, très souvent, il devrait être possible de concilier des travaux d'entretien ou d'amélioration de chenaux d'accès et de zones portuaires ensablées avec des réalimentations de plages, la plus-value des dragages n'intervenant que dans les installations de rejet ou d'augmentation des parcours des dragues. Comparativement à la déperdition de notre patrimoine littoral, voire à son exploitation sur le plan touristique, ce coût supplémentaire reste très faible surtout si l'on se rappelle que, chaque année, $800 \mathrm{~km}$ de nos côtes reculent de plus d'un mètre.

\section{Références}

[1] Migniot C. et Viguier J. 1979. Influence de l'extraction de granulat en mer sur l'équilibre du littoral. La Houille blanche, 3 .
[2] Migniot C. 1982. Etude de la dynamique sédimentaire marine, fluviale, estuarienne. Thèse de doctorat d'Etat, Université de Paris sud.

[3] SHORE PROTECTION MANUAL. 1984. 4ème édition US Army, Coastal Engineering Research Center.

[4] Manoujian S. et Migniot C. 1979. Un exemple de difficulté de la protection du littoral contre l'érosion marine. La Houille Blanche, 4/5.

[5] Manoujian S. 1983. Rechargement des plages à l'aide de produits de dragages ou prélevés au large. Le cas de la Côte d'Anglet, du littoral d'Agadir et de la plage de Knokke. Congrès Erosion et défense des côtes, ASTEO.

[6] Manoujian S. 1984. Protection du littoral d'Anglet contre l'érosion marine : Impact des travaux réalisés au cours des dix dernières années. 18èmes Journées de l'Hydraulique.

[7] Lalaut Y. et Manoujian S. 1986. Enquête sur les ouvrages de protection d'un littoral sableux existants sur tout le littoral français, STC.

[8] LCHF. 1986. Protection contre les érosions du littoral sud de Cap Breton

[9] LCHF, SOGREAH. 1990. Etude bibliographique sur le rechargement des plages par les produits de dragage. STC. 